\title{
Detecting Depolarized Targets using a New Geometrical Perturbation Filter
}

\author{
Armando Marino, Member, IEEE, Shane R. Cloude, Fellow, IEEE, and Iain H. Woodhouse, Member, \\ IEEE.
}

\begin{abstract}
Target detectors using polarimetry are often focused on single targets, since these can be characterized in a simpler and deterministic way. The algorithm proposed in this paper is aimed at the more difficult problem of partial target detection (i.e. targets with arbitrary degree of polarization). The authors have already proposed a single target detector employing filters based on a geometrical perturbation. In order to enhance the algorithm to the detection of partial targets, a new vector formalism is introduced. The latter is similar to the one exploited for single targets but suitable for complete characterization of partial targets. A new feature vector is generated starting from the covariance matrix, and exploited for the perturbation method. Validation against L-band fully polarimetric airborne $E$ SAR, and satellite ALOS-PALSAR data and X-band dual polarimetric TerraSAR-X data is provided with significant agreement with the expected results. Additionally, a comparison with the supervised Wishart classifier is presented revealing improvements.
\end{abstract}

Index Terms - Synthetic Aperture Radar (SAR), Polarimetry, Target Detection, Classification.

\section{INTRODUCTION}

$\mathrm{T}$ HE polarization of the electromagnetic field scattered by an object keeps valuable information about the scatterer and this can be exploited to detect or classify specific targets. The possible applications are several, from surveillance or land use monitoring to biophysical parameter extraction [1-4]. In polarimetry, one of the most fundamental distinctions among scatterers is between single and partial targets. A single target scatters a stable (and deterministic) polarization state and it can be completely described with a single scattering (Sinclair) matrix or equivalently a scattering vector, defined in (1):

$\underline{k}=\frac{1}{2} \operatorname{Trace}([S] \Psi)=\left[k_{1}, k_{2}, k_{3}, k_{4}\right]^{T}$,

Armando Marino is with the ETH Zürich, Institute of Environmental Engineering (IfU-Baug), Schafmattstr. 6, CH-8093, Zürich, Switzerland (phone: +41/(0)44-633-3404; e-mail: marino@ifu.baug.ethz.ch). Shane R. Cloude is with AEL Consultants, 26 Westfield Avenue, Cupar, Fife, KY15 5AA, Scotland, UK (e-mail: aelc@mac.com). Iain H. Woodhouse is with the University of Edinburgh, School of GeoSciences, Edinburgh, EH8 9XP (email: i.h.woodhouse@ed.ac.uk).

This work was partially fund by the UK DSTL (in the framework of the SARTOM project run by eOsphere Ltd) and by the ESA DRAGON-2 project number 5344 (run by the University of Rennes, France). where $[S]$ is the scattering matrix, Trace(.) is the sum of the diagonal elements of the matrix inside, and $\Psi$ is a complete set of $2 \times 2$ basis matrices under a Hermitian inner product [1, $2,5]$. In case of a reciprocal medium and monostatic sensor, $\underline{k}$ is a three dimensional complex vector. The scattering vector representation was revealed more advantageous than the scattering matrix for the detector proposed in this paper since it works with vectors in a linear geometric space (3 dimensional complex, $S U(3)[6,7])$ where inner products are easier to define. Finally, the scattering mechanism is defined as a normalized vector shown in (2)

$\underline{\omega}=\underline{k} /|\underline{k}|$.

On the other hand, targets observed by a SAR system are generally not idealized single scattering targets, but a combination of different targets inside the same resolution cell, which we refer to as a partial target [8]. The latter can be modeled as a stochastic process, consequently a single scattering matrix is not sufficient for its complete description and the second order statistics must be extracted. The target covariance matrix can be estimated as shown in (3):

$[C]=\left\langle\underline{k} \underline{k}^{* T}\right\rangle$,

where $\langle$.$\rangle is the finite averaging operator. In general, if the$ scattering vector (in a generic basis) is $\underline{k}=\left[k_{1}, k_{2}, k_{3}\right]^{T}$, with $k_{1}, k_{2}$ and $k_{3}$ complex numbers, the covariance matrix will be

$[C]=\left[\begin{array}{ccc}\left\langle\left|k_{1}\right|^{2}\right\rangle & \left\langle k_{1} k_{2}^{*}\right\rangle & \left\langle k_{1} k_{3}^{*}\right\rangle \\ \left\langle k_{2} k_{1}^{*}\right\rangle & \left\langle\left|k_{2}\right|^{2}\right\rangle & \left\langle k_{2} k_{3}^{*}\right\rangle \\ \left\langle k_{3} k_{1}^{*}\right\rangle & \left\langle k_{3} k_{2}^{*}\right\rangle & \left\langle\left|k_{3}\right|^{2}\right\rangle\end{array}\right]$.

The methodology proposed in this paper takes advantage of the polarimetric coherence [1, 2]. If two scattering mechanisms $\underline{\omega}_{1}$ and $\underline{\omega}_{2}$ are considered, the polarimetric coherence is

$$
\gamma=\frac{\left\langle i\left(\underline{\omega}_{1}\right) i^{*}\left(\underline{\omega}_{2}\right)\right\rangle}{\sqrt{\left\langle i\left(\underline{\omega}_{1}\right) i^{*}\left(\underline{\omega}_{1}\right)\right\rangle\left\langle i\left(\underline{\omega}_{2}\right) i^{*}\left(\underline{\omega}_{2}\right)\right\rangle}},
$$

where $i$ is the image evaluated as 
$i\left(\omega_{j}\right)=\underline{\omega}_{j}^{* T} \underline{k}$ with $j=1,2$.

Or in terms of the target covariance matrix [1]:

$$
\gamma=\frac{\underline{\omega}_{1}^{{ }^{T}}[C] \underline{\omega}_{2}}{\sqrt{\left(\underline{\omega}_{1}^{* T}[C] \underline{\omega}_{1}\right)\left(\underline{\omega}_{2}^{{ }^{T}}[C] \underline{\omega}_{2}\right)}} .
$$

Our objective in this paper is to design a detector using this coherence.

In this paper, we propose a new partial target detector, which can be easily evolved into a land cover classifier. In the literature, the issue of detection and classification exploiting radar polarimetry is extensively treated. Two main methodologies can be delineated. The first one lists a series of algorithms based on physical approaches. For instance, several coherent and incoherent target decompositions were proposed $[1,2,8]$. The other family of algorithms exploits the statistics of the scattering process [9-13]. The most common classifier considers a maximum likelihood (ML) of the covariance matrix modeled as a Wishart probability distribution [14]. The classification performance can be substantially enhanced by adding more information and exploiting multi-frequencies or interferometric data. In the literature, many examples of the benefits provided by these methodologies are reported [3, 4, 15-18].

The paper is structured as follows, in section II we first review the structure of the single target detector already published in [19-21]. We then develop an important geometrical interpretation of the filter which allows us to develop in section III a generalized form of the detector for depolarizing targets. In section IV we then show how this detector can be used to generate a supervised and unsupervised classifier before considering in section $\mathrm{V}$ its application to air and space borne radar data sets.

\section{Single TARGET DETECTOR}

\section{A. Physical derivation}

A single target detector was already developed and published by the authors in [19-21]. It can be summarized as follows:

given a scattering mechanism $\underline{\omega}_{T}$ proportional to the target to be detected, and given a second scattering mechanism $\underline{\omega}_{P}$ close to $\underline{\omega}_{T}$ within the target space, the polarimetric coherence between the images formed with $\underline{\omega}_{P}$ close to $\underline{\omega}_{T}$ is high if in the averaging window the component of interest (proportional to $\underline{\omega}_{T}$ ) is stronger than the other two orthogonal components.

First step is to define a basis for the target space where the target of interest lies exclusively on one component of the 3 dimensional complex vector $\underline{k}$. This operation is always possible and requires the multiplication by a unitary matrix [1]. In the following, the scattering mechanism after the change of basis is therefore always regarded as
$\underline{\omega}_{T}=[1,0,0]^{T}$. The covariance matrix $[C]$ will be calculated starting from this basis. The resulting image when the target $\underline{\omega}_{T}$ is selected is

$$
i\left(\underline{\omega}_{T}\right)=k_{1} \text {. }
$$

In (8), the second and third components of the scattering vector (i.e. $k_{2}$ and $k_{3}$ ) disappear, since the target of interest lies exclusively in $k_{1}$. For this reason, $k_{2}$ and $k_{3}$ are considered as clutter.

The second scattering mechanism $\underline{\omega}_{P}$ (i.e. perturbed target) is obtained by rotating slightly the vector $\underline{\omega}_{T}$ in the polarimetric space. In a first attempt, the rotation can be accomplished using the Huynen parameters [22] or the $\alpha$ angle parameterization $[1,2,23]$ (the procedure is explained more thoroughly in [19-21]). Having obtained the expression for the perturbed target in the basis exploited by the parameterization, the same change of basis that makes $\underline{\omega}_{T}=[1,0,0]^{T}$ must be performed on $\underline{\omega}_{P}$. Consequently, $\underline{\omega}_{P}=[a, b, c]^{T}$, with $a, b$ and $c$ complex numbers. Considering $\underline{\omega}_{T} \approx \underline{\omega}_{P}$, we have $|a| \approx 1,|b| \approx 0$ and $|c| \approx 0$.

The polarimetric coherence can then be estimated as

$\gamma\left(\underline{\omega}_{T}, \underline{\omega}_{P}\right)=\frac{\left\langle i\left(\underline{\omega}_{T}\right) i^{*}\left(\underline{\omega}_{P}\right)\right\rangle}{\sqrt{\left\langle i\left(\underline{\omega}_{T}\right) i^{*}\left(\underline{\omega}_{T}\right)\right\rangle\left\langle i\left(\underline{\omega}_{P}\right) i^{*}\left(\underline{\omega}_{P}\right)\right\rangle}}$,

where:

$\left\langle i\left(\underline{\omega}_{T}\right) i^{*}\left(\underline{\omega}_{P}\right)\right\rangle=a\left\langle\left|k_{1}\right|^{2}\right\rangle+b\left\langle k_{1} k_{2}^{*}\right\rangle+c\left\langle k_{1} k_{3}^{*}\right\rangle$,

$\left\langle i\left(\underline{\omega}_{T}\right) i^{*}\left(\underline{\omega}_{T}\right)\right\rangle=\left\langle\left|k_{1}\right|^{2}\right\rangle$,

$\left\langle i\left(\underline{\omega}_{P}\right) i^{*}\left(\underline{\omega}_{P}\right)\right\rangle=|a|^{2}\left\langle\left|k_{1}\right|^{2}\right\rangle+|b|^{2}\left\langle\left|k_{2}\right|^{2}\right\rangle+|c|^{2}\left\langle\left|k_{3}\right|^{2}\right\rangle+$

$+2 \operatorname{Re}\left(a b^{*}\left\langle k_{1} k_{2}^{*}\right\rangle\right)+2 \operatorname{Re}\left(a c^{*}\left\langle k_{1} k_{3}^{*}\right\rangle\right)+2 \operatorname{Re}\left(c b^{*}\left\langle k_{3} k_{2}^{*}\right\rangle\right)$.

Finally, the detector is obtained setting a threshold on the coherence amplitude:

$\left|\gamma\left(\underline{\omega}_{T}, \underline{\omega}_{P}\right)\right| \geq T$,

where $T$ is a threshold. In (11) the phase of the coherence $\gamma$ does not seem to have any clear physical interpretation. Therefore, the proposed algorithm is currently focused on the exploitation of the amplitude alone. However, the authors leave as future work the analysis of the phase. Unfortunately, $|\gamma|$ cannot be used as a detector, since the cross products between the components of the scattering vector introduce biases, in case the components are correlated with each other [19-21]. For instance, the correlation can be introduced by a single target which has projection over both target and clutter components. Geometrically, the latter can be any single target 
which does not lie or is orthogonal to the complex plane spanned by the two clutter components. In order to remove the bias, the cross products must be neglected. The polarimetric coherence operator is substituted with another operator working on the space of the target components power, as shown in (12-14):

$$
\begin{aligned}
& \gamma_{d}\left(\underline{\omega}_{T}, \underline{\omega}_{P}\right)=\frac{\left|\underline{\omega}_{T}^{{ }^{*} T}[P] \underline{\omega}_{P}\right|}{\sqrt{\left(\underline{\omega}_{T}^{*_{T} T}[P] \underline{\omega}_{T}\right)\left(\underline{\omega}_{P}^{* T}[P] \underline{\omega}_{P}\right)}}, \\
& \text { where: }[P]=\left[\begin{array}{ccc}
\left\langle\left|k_{1}\right|^{2}\right\rangle & 0 & 0 \\
0 & \left\langle\left|k_{2}\right|^{2}\right\rangle & 0 \\
0 & 0 & \left\langle\left|k_{3}\right|^{2}\right\rangle
\end{array}\right], \\
& \text { or }[P]=\operatorname{diag}\left(\left\langle\left|k_{1}\right|^{2}\right\rangle,\left\langle\left|k_{2}\right|^{2}\right\rangle,\left\langle\left|k_{3}\right|^{2}\right\rangle\right) .
\end{aligned}
$$

The modified coherence amplitude in (12) will be regarded as the detector. The latter is dependent exclusively on the power components of the scattering vector $\underline{k}$.

$$
\gamma_{d}=\frac{|a|\left\langle\left|k_{1}\right|^{2}\right\rangle}{\sqrt{\left\langle\left|k_{1}\right|^{2}\right\rangle\left(|a|^{2}\left\langle\left|k_{1}\right|^{2}\right\rangle+|b|^{2}\left\langle\left|k_{2}\right|^{2}\right\rangle+|c|^{2}\left\langle\left|k_{3}\right|^{2}\right\rangle\right)}} .
$$

After dividing both numerator and denominator by $|a|\left\langle\left|k_{1}\right|^{2}\right\rangle$, the amplitude of the polarimetric coherence becomes:

$$
\gamma_{d}=\frac{1}{\sqrt{1+\frac{|b|^{2}}{|a|^{2}} \frac{\left\langle\left|k_{2}\right|^{2}\right\rangle}{\left\langle\left|k_{1}\right|^{2}\right\rangle}+\frac{|c|^{2}}{|a|^{2}} \frac{\left\langle\left. k_{3}\right|^{2}\right\rangle}{\left\langle\left|k_{1}\right|^{2}\right\rangle}}}
$$

If the powers are defined as $P_{T}=\left\langle\left|k_{1}\right|^{2}\right\rangle, P_{C 2}=\left\langle\left|k_{2}\right|^{2}\right\rangle$, $P_{C 3}=\left\langle\left|k_{3}\right|^{2}\right\rangle$, the expression of the detector can be simplified:

$$
\gamma_{d}=\frac{1}{\sqrt{1+\frac{|b|^{2}}{|a|^{2}} \frac{P_{C 2}}{P_{T}}+\frac{|c|^{2}}{|a|^{2}} \frac{P_{C 3}}{P_{T}}}} .
$$

We regard to $(|b| /|a|)^{2}$ and $(|c| /|a|)^{2}$ as Reduction Ratios $($ RedR). The perturbed targets are chosen in order to have small RedR. Looking at (17), the lowering effect played by the $R e d R$ is clear. If the clutter powers are lower than the target power the two terms on the denominator are negligible and $\gamma_{d}=1$.

The final expression of the detector sets a threshold on (17): $\gamma_{d}\left(\underline{\omega}_{T}, \underline{\omega}_{P}\right) \geq T$.

In [19-21], the optimization of the parameters and the threshold selection are treated. In particular, the optimal choice (i.e. it does not present biases) of the perturbed target in absence of a priori information about clutter is $|b|=|c|$. If the total power of the clutter is indicated with $P_{C}=P_{C 2}+P_{C 3}$ the detector is further simplified:

$$
\gamma_{d}=\frac{1}{\sqrt{1+\operatorname{RedR} \frac{P_{C}}{P_{T}}}} .
$$

The detector derivation is based on the scattering vector formalism and the possibility to describe a single target with a three dimensional complex vector. On the other hand, partial targets need a wider algebraic space (i.e. with more dimensions). In order to proceed in the development and extend the detector to partial targets, a useful geometrical generalization must be provided, which will allow the extension to a higher dimensional space.

\section{B. Geometrical interpretation}

In this section, a new geometrical interpretation of the polarimetric detector will be provided. Given a vector $\underline{x}$ in the target space $(S U(3))$, a linear transformation can be defined as $[A] \underline{x}=\underline{b}$,

where $[A]$ is a $3 \times 3$ matrix (in general it can be any $3 \times N$ matrix). $[A]$ is a transformation of the vector $\underline{x}$ into a resulting vector $\underline{b}$, which lies in the subspace spanned by the columns of $[A]$ (or its null subspace) $[24,25]$. If $[A]$ is a diagonal matrix the columns of $[A]$ will always represent a basis for the entire $\square^{3}$ space (as long as all the elements of the diagonal are different from zero). In particular, if $[A]=[I]$ the transformation is from the entire space to the entire space using the same ortho-normal basis. Clearly, this transformation leads to $\underline{b}=\underline{x}$. In case $[A]$ is a diagonal matrix with at least one element different from 1 , the basis used is not the ortho-normal one (i.e. the axis are not normalized).

The matrix $[A]$ can be generated as $[A]=\operatorname{diag}\left(k_{1}, k_{2}, k_{3}\right)$, where again $\underline{k}=\left[k_{1}, k_{2}, k_{3}\right]^{T}$ is the scattering vector in the basis which makes $\underline{\omega}_{T}=[1,0,0]^{T}$. If the coordinate basis is defined as $\underline{e}_{1}=[1,0,0]^{T}, \underline{e}_{2}=[0,1,0]^{T}$ and $\underline{e}_{3}=[0,0,1]^{T}$ the transformed vector $\underline{b}$ is

$\underline{b}=x_{1} k_{1} \cdot \underline{e}_{1}+x_{2} k_{2} \cdot \underline{e}_{2}+x_{3} k_{3} \cdot \underline{e}_{3}$,

where $\underline{x}=\left[x_{1}, x_{2}, x_{3}\right]^{T}$. Therefore, $[A] \underline{x}=\underline{b}$ can be interpreted as a weighting of the components of $\underline{x}$, where the weights are the diagonal elements of $[A]$. The metric of the space will be clearly redefined and all the vectors will be stretched along a preferential axis.

The standard Euclidean inner product between $\underline{\omega}_{T}$ and $\underline{\omega}_{P}$ can be written as $\underline{\omega}_{T}^{{ }^{*} T} \underline{\omega}_{P}[24,25]$. The weighting of the scattering mechanism can be accomplished with

\section{$[A] \underline{\omega}_{T}=\underline{b}_{T}$ and $[A] \underline{\omega}_{P}=\underline{b}_{P}$.}

The detector is based on the calculation of an inner product 
between target and perturbed target in a basis set which amplifies the direction represented by the observed target. Clearly, the inner product $\underline{b}_{T}^{* T} \underline{b}_{P}$ cannot be calculated pixel by pixel, since the pixel statistical variation (i.e. speckle) can result in improper estimation of the actual observed target [2629]. The average over independent realizations is required to obtain reliable results. Therefore, the inner product $\underline{b}_{T}^{* T} \underline{b}_{P}$ is substituted with the averaged one

$$
\begin{aligned}
\left\langle\underline{b}_{T}^{{ }^{* T}} \underline{b}_{P}\right\rangle & =\left\langle\left([A] \underline{\omega}_{T}\right)^{*_{T}}\left([A] \underline{\omega}_{P}\right)\right\rangle= \\
& =\underline{\omega}_{T}^{* T}\left\langle[A]^{* T}[A]\right\rangle \underline{\omega}_{P}= \\
& =\underline{\omega}_{T}^{* T}[P] \underline{\omega}_{P},
\end{aligned}
$$

where again $[P]=\operatorname{diag}\left(\left\langle\left|k_{1}\right|^{2}\right\rangle,\left\langle\left|k_{2}\right|^{2}\right\rangle,\left\langle\left|k_{3}\right|^{2}\right\rangle\right)$. Please note, the expression of $[P]$ is exactly equivalent to the one obtained in (13).

Last step is the normalization of the weighted inner product:

$\gamma_{d}=\frac{\left|\underline{\omega}_{T}^{{ }^{*} T}[P] \underline{\omega}_{P}\right|}{\sqrt{\left(\underline{\omega}_{T}^{{ }^{T} T}[P] \underline{\omega}_{T}\right)\left(\underline{\omega}_{P}^{{ }^{T} T}[P] \underline{\omega}_{P}\right)}}$.

The latter represents the same expression obtained with the physical approach.

Now, we want to address the question: why the weighted inner product results in a detector? When the standard normalized inner product between $\underline{\omega}_{T}$ and $\underline{\omega}_{P}$ is estimated, the correlation (which increases the value of the coherence) is introduced solely by the first component. The second and third components cannot be correlated since $\underline{\omega}_{T}$ has only one nonzero component. Specifically, the amplitude of the correlation is equal to the cosine of the angle $\phi$ between the two vectors (since they are normalized) [24, 25]:

$\left|\underline{\omega}_{T}^{{ }^{*} T} \underline{\omega}_{P}\right|=\cos \phi=|a|$.

Since the first component is the only one bringing correlation, the inner product varies on the base of the amount of weight allocated to the first component (compared to the others). Generally, the weighting has two main effects on the scattering mechanisms: a rotation and a rescaling. The rescaling can be neglected since the inner product is subsequently normalized. On the other hand, the rotation affects only $\underline{\omega}_{P}$, because $\underline{\omega}_{T}$ cannot change direction and it will always be along the first component,

$\forall[A], \underline{b}_{T}=[A] \underline{\omega}_{T}=k_{1} \underline{\omega}_{T}$.

The perturbed target becomes:

$b_{p}=[A] \underline{\omega}_{P}=\left[k_{1} a, k_{2} b, k_{3} c\right]^{T}=k_{1} a \underline{\omega}_{T}+k_{2} b \underline{\omega}_{C 2}+k_{2} c \underline{\omega}_{C 3}$,

where $\underline{\omega}_{C 2}=[0,1,0]^{T}$ and $\underline{\omega}_{C 3}=[0,0,1]^{T}$.

In conclusion, if the rotation makes the resulting vector $\underline{b}_{P}$ closer to $\underline{b}_{T}$ the angle between them reduces and the coherence increases. By definition, the normalized inner product between the weighted scattering mechanisms is the detector, consequently the angle between $\underline{b}_{P}$ and $\underline{b}_{T}$ becomes $\widehat{\vartheta}=\cos ^{-1}\left(\gamma_{d}\right)$. This angle decreases after the weighting if

$\widehat{\vartheta}=\cos ^{-1}\left(\gamma_{d}\right)<\cos ^{-1}(|a|)=\phi$,

$\gamma_{d}>|a|$.

Geometrically, this occurs when the observed target has a $k_{1}$ component stronger than the others. In other words, the correlation increases if $\underline{\omega}_{P}$ is stretched toward a direction where the $k_{1}$ component is stronger.

Clearly, the fact that the angle is reduced is not sufficient to guarantee detection, since the coherence $\gamma_{d}$ is required to be over the threshold as well. We can now use this idea to construct a new detector for depolarizing targets.

\section{Partial target Detector}

\section{A. Formulation}

In order to extend the detectability of the algorithm to partial targets, a new formalism similar to the one used for single targets must first be introduced. To this end, a feature partial scattering vector is defined:

$$
\begin{aligned}
& \underline{t}=\operatorname{Trace}([C] \Psi)=\left[t_{1}, t_{2}, t_{3}, t_{4}, t_{5}, t_{6}\right]^{T}= \\
& =\left[\left\langle\left|k_{1}\right|^{2}\right\rangle,\left\langle\left|k_{2}\right|^{2}\right\rangle,\left\langle\left|k_{3}\right|^{2}\right\rangle,\left\langle k_{1}^{*} k_{2}\right\rangle,\left\langle k_{1}^{*} k_{3}\right\rangle,\left\langle k_{2}^{*} k_{3}\right\rangle\right]^{T} .
\end{aligned}
$$

where $\Psi$ is a set of $6 \times 6$ basis matrices under a Hermitian inner product. The $\underline{t}$ vector lies in a subspace of $\square^{6}$ (it is closed for sum and scalar multiplication and includes the zero). In particular, the first three components are real positive and the second three complex. To have physical feasibility the last three elements must obey the Cauchy-Schwarz [24] inequality, that always happens since $[C]$ is a covariance (positive semi-definite) matrix

$\langle|\underline{x}|\rangle\langle|\underline{y}|\rangle \geq\left|\left\langle\underline{x}^{{ }^{*}} \underline{y}\right\rangle\right|:$

$\sqrt{t_{1} t_{2}} \geq\left|t_{4}\right|, \sqrt{t_{1} t_{3}} \geq\left|t_{5}\right|, \sqrt{t_{2} t_{3}} \geq\left|t_{6}\right|$.

Any physically realizable $\underline{t}$ represents completely and uniquely a partial target. In particular, the partial target to be detected and the perturbed target are regarded as

$\hat{t}_{T}=\operatorname{Trace}\left(\left[C_{T}\right] \Psi\right) /\left\|\operatorname{Trace}\left(\left[C_{T}\right] \Psi\right)\right\|$,

$\hat{t}_{P}=\operatorname{Trace}\left(\left[C_{P}\right] \Psi\right) /\left\|\operatorname{Trace}\left(\left[C_{P}\right] \Psi\right)\right\|$.

The latter could be seen as the equivalent of the scattering mechanisms for partial targets. Although the optimization of the perturbation has mathematical foundations [19-21], physical meaning can be attributed to the process. For 
instance, the covariance matrix for the target $\left[C_{T}\right]$ can be mapped into a Kennaugh matrix $\left[K_{T}\right][1]$. Subsequently, the Huynen transformations can be performed on the Kennaugh matrix generating a slightly different target $\left[K_{P}\right][22]$. Finally, the perturbed Kennaugh matrix $\left[K_{P}\right]$ is mapped back into a covariance matrix $\left[C_{P}\right]$ (and the vector $\hat{t}_{P}$ ). The latter is merely an example of physical perturbation of the partial target and any other parameterization can be exploited.

Again, a change of basis is performed which makes the target of interest lie only in one nonzero component:

$\hat{t}_{T}=[1,0,0,0,0,0]^{T}$ and $\hat{t}_{P}=[a, b, c, d, e, f]^{T}$.

In case the perturbation is performed without any physical model, $\hat{t}_{P}$ must be selected preserving the physical feasibility:

$$
\begin{aligned}
& a, b, c \in \square^{+}, \\
& \sqrt{a b} \geq|d|, \sqrt{a c} \geq|e|, \sqrt{b c} \geq|f|, \\
& a^{2}+b^{2}+c^{2}+|d|^{2}+|e|^{2}+|f|^{2}=1 .
\end{aligned}
$$

Additionally, by definition of perturbed target:

$$
a \gg b, a>c, a>|d|, a>>|e|, a>>|f| \text {. }
$$

The elements on the diagonal of $[A]$ are the components of the partial scattering vector $\underline{t}$ after the change of basis which makes $\hat{t}_{T}=[1,0,0,0,0,0]^{T}$. The change of basis can be achieved by multiplying by a unitary matrix, where the columns can be derived by solving a linear equation system, where the unknowns are 5 rotation angles and 5 phase angles.

A simpler way to generate $[A]$ considers a Gram-Schmidt ortho-normalization $(G S)$ in $\square^{6}$, where the first axis is the vector $\hat{t}_{T}$. The components of $[A]$ are calculated with the inner product of the basis for the observable $\underline{t}$. If $\underline{u}_{1}=\underline{t}_{T}$, $\underline{u}_{2}, \underline{u}_{3}, \underline{u}_{4}, \underline{u}_{5}$ and $\underline{u}_{6}$ represent the ortho-normal basis then

$$
[A]=\operatorname{diag}\left(\underline{\hat{t}}_{T}^{* T} \underline{t}^{*} \underline{u}_{2}^{{ }^{*} T} \underline{t}, \underline{u}_{3}^{* T} \underline{t}, \underline{u}_{4}^{* T} \underline{t}, \underline{u}_{5}^{* T} \underline{t}, \underline{u}_{6}^{* T} \underline{t}\right) \text {. }
$$

The detector can be achieved with

$$
\left([A] \underline{\hat{t}}_{T}\right)^{* T}\left([A] \underline{\hat{t}}_{P}\right)=\underline{\hat{t}}_{T}^{* T}[A]^{* T}[A] \underline{\hat{t}}_{P}=\underline{\hat{t}}_{T}^{* T}[P] \hat{t}_{P},
$$

where $[P]=\operatorname{diag}\left(P_{1}, P_{2}, P_{3}, P_{4}, P_{5}, P_{6}\right)$.

$$
\gamma_{d}\left(\underline{t}_{T}, \underline{t}_{P}\right)=\frac{\underline{t}_{T}^{*_{T}}[P] \underline{t}_{P}}{\sqrt{\left(\underline{t}_{T}^{*_{T}}[P] \underline{t}_{T}\right)\left(\underline{t}_{P}^{* T}[P] \underline{t}_{P}\right)}},
$$

$$
\gamma_{d}=\frac{1}{\sqrt{1+\frac{b^{2}}{a^{2}} \frac{P_{2}}{P_{1}}+\frac{c^{2}}{a^{2}} \frac{P_{3}}{P_{1}}+\frac{|d|^{2}}{a^{2}} \frac{P_{4}}{P_{1}}+\frac{|e|^{2}}{a^{2}} \frac{P_{5}}{P_{1}}+\frac{|f|^{2}}{a^{2}} \frac{P_{6}}{P_{1}}}} .
$$

The partial detector is formally similar to the single one in
(17) (except for the number of terms), consequently all the mathematical optimizations performed for the single target detector can be adopted here [19-21]. Specifically, in absence of a priori information about the clutter, the perturbed target is chosen as

$b=c=|d|=|e|=|f|$.

If we define the clutter as $P_{c}=P_{2}+P_{3}+P_{4}+P_{5}+P_{6}$, the target as $P_{1}=P_{T}$ and $\operatorname{RedR}=(b / a)^{2}$ the detector becomes

$$
\gamma_{d}=\frac{1}{\sqrt{1+\operatorname{RedR} \frac{P_{C}}{P_{T}}}}
$$

The detector is finalized with a threshold $T$ on $\gamma_{d}$. The result of the algorithm, here referred to as detection mask, is zero if the detector is under the threshold or equal to the detector if it is above the threshold. In other words:

$\left\{\begin{array}{c}m(x, y)=0 \quad \text { if } \gamma_{d}(x, y)<T \\ m(x, y)=\gamma_{d}(x, y) \quad \text { if } \gamma_{d}(x, y) \geq T,\end{array}\right.$

where $m$ is the image mask, $(x, y)$ represents the coordinate of a generic pixel. Using this typology of mask (and not a 1 or 0 binary format), we want to preserve information about the dominance of the target in the cell. This will be useful for the design of a classifier as we show in section IV.

\section{B. Physical feasibility}

In this section, clarifications about the uniqueness and the Gram-Schmidt ortho-normalization $(G S)$ are provided.

The former is guaranteed since, by definition, any partial target can be described by a covariance matrix $[C]$ (specifically, 9 real independent parameters). Additionally, all the independent elements of $[C]$ are unequally mapped in the feature vector $\underline{t}$. In the proposed 6 dimensional complex space, any partial target can be uniquely related to a single feature vector $\underline{t}$, independently on the target degree of polarization: from pure (single targets) to completely unpolarized (random noise). In conclusion, there is a 1 by 1 relationship between the physically feasible $\underline{t}$ and any partial target.

Regarding the GS, generally, the resulting basis does not represent a set of physical feasible targets, except for the first axis, which is calculated starting from a physical realizable vector $\hat{t}_{T}$. GS generates a basis for $\square^{6}$ but not all the vectors of $\square^{6}$ are physically feasible. This does not however represent a limitation of the detector. The axes $\underline{u}_{2}, \underline{u}_{3}, \underline{u}_{4}$, $\underline{u}_{5}$ and $\underline{u}_{6}$, obtained with the $G S$ ortho-normalization, span a subspace of $\square^{6}$ which is completely orthogonal to the first axis $\underline{t}_{T}$ (i.e. the orthogonal complement of $\hat{t}_{T}$ in $\square^{6}$ ). This means that given a vector 
$\underline{u}=c_{2} \cdot \underline{u}_{2}+c_{3} \cdot \underline{u}_{3}+c_{4} \cdot \underline{u}_{4}+c_{5} \cdot \underline{u}_{5}+c_{6} \cdot \underline{u}_{6}$,

we have

$\underline{u}_{1}=\underline{\hat{t}}_{T} \perp \underline{u}, \forall c_{2}, c_{3}, c_{4}, c_{5}, c_{6} \in C$.

The first vector of the GS basis $\underline{u}_{1}$ is always physically realizable, since it is equal to $\hat{t}_{T}$ (i.e. the target to be detected). We refer to the orthogonal complement subspace of $\hat{t}_{T}$ in $\square^{6}$ as $\mathrm{Z}$. Clearly only a portion (i.e. subspace) of $\mathrm{Z}$ represents physically feasible targets. Moreover, a physically feasible target extracted from the data, will generally have a component in the $\mathrm{Z}$ subspace, called $\underline{z}$. The length of $\underline{z}$ is independent of the basis used to represent $\mathrm{Z}$ (since the length is an invariant property of the vector $z$ ) $[24,25]$. Therefore, we do not require that $\underline{u}_{2}, \underline{u}_{3}, \underline{u}_{4}, \underline{u}_{5}$ and $\underline{u}_{6}$ are physically feasible vectors, as long as they represent a basis for $\mathrm{Z}$.

As (42) shows, we are interested in $P_{T}$ while $P_{C}$ represent the rest of the power. Clearly, equal results are obtained starting from (42) and considering $P_{C}=P_{t o t}-P_{T}$, where

$P_{\text {tot }}=\underline{t}^{* T} \underline{t}$

is the total power of $\underline{t}$ evaluated in the original basis. The final simplified expression of the detector is

$$
\gamma_{d}=\frac{1}{\sqrt{1+\operatorname{RedR}\left(\frac{P_{t o t}}{P_{T}}-1\right)}}=\frac{1}{\sqrt{1+\frac{b^{2}}{1-5 b^{2}}\left(\frac{\underline{t}^{* T} \underline{t}}{\left|\underline{t}^{* T} \hat{t}_{T}\right|^{2}}-1\right)}} \geq T
$$

Summarizing, the detector obtained with the projections on the $G S$ basis and the one with the total power are entirely equivalent when $b=c=|d|=|e|=|f|$ (i.e. absence of $a$ priori information about clutter), since $b, c,|d|,|e|$ and $|f|$ can be collected, and the expression of $P_{C}$ can be substituted.

\section{Parameter selection}

The partial target detector proposed in this paper shares the same mathematical formalism of the single target detector in [19-21]. As a consequence, all the mathematical optimizations can be extended to this case. For the sake of brevity, we only present the selection of threshold and RedR. This can be accomplished starting from a dispersion equation based on the angular distance between the observed partial target and the one of interest.

After some algebraic manipulation of (42) and substituting $S C R=\frac{P_{T}}{P_{C}} \Rightarrow \frac{P_{t o t}}{P_{T}}-1=\frac{1}{S C R}$, we can find the dispersion expression:

$$
0 \leq \frac{P_{C}}{P_{T}}=\frac{1}{S C R} \leq \frac{1}{\operatorname{RedR}}\left(\frac{1}{T^{2}}-1\right) .
$$

The first inequality is consequence of the fact that the power of the clutter cannot be bigger than the total power.
Equation 48 exhibits a relationship among Signal to Clutter Ratio $(S C R)$, threshold and RedR. Here, the SCR has a slightly alternative geometrical interpretation compared with classical detection. In general, it represents the ratio between the power of target and clutter located in the scene. Now, this ratio corresponds to a measure of the angular distance between the observed vector (i.e. target) and the one of interest. Its selection conforms to selectivity requirements of the detector and it can be related to the target properties. On real data, the extraction of the second order statistics (and consequently the characterization of a partial target) is not ideal since a finite averaging is needed and the target of interest may not be completely homogeneous. For this reason, the extracted $\underline{t}$ vector of a real target still presents statistical variation and could be seen as a random variable. In general, when the target of interest is expected to be polarimetricaly stable, a higher $S C R$ can be utilized, leading to a smaller false alarm rate. With polarimetricaly stable we mean that the angular distance of its $\underline{t}$ vector instances (realizations) is small (i.e. the representation of the target is stable over all the scene). However, if the target is anticipated to change slightly over the entire scene, a smaller $S C R$ is to be preferred, which leads to higher probability of detection. In the following experiments, the $S C R$ for detections is chosen equal to 50, since this value seems to provide the best compromise between probability of detection and false alarm. However, common values can go from 2 to 100 .

Having defined the $S C R$, two unknowns remain in (48). Therefore, one unknown can be expressed as function of the other. Equation 49 presents one of the two possible solutions of (48) when the equality sign is substituted:

$\operatorname{RedR}=S C R /\left(\frac{1}{T^{2}}-1\right)$.

The threshold can be freely set. In the following experiments $T=0.98$, although any other values smaller than 1 could be theoretically employed. However, a relatively high value of $T$ entails a smaller variance of the polarimetric coherence, which increases the statistical performances of the detector.

Once selected $T$, the last parameter (i.e. RedR) can be set. In our experiments, $\operatorname{Red} R=1.85$.

\section{Dual polarimetric detection}

This final section is dedicated to the use of dual polarimetric data. The proposed algorithm is based on a geometrical operation which is theoretically independent on the dimensions of the space considered, as long as it is Euclidean. Consequently, it can be exported to any Euclidean vector space. The demand of quad polarimetric data is a physical requirement, since the acquisition of all the elements of the scattering matrix is needed to characterize uniquely a generic depolarized target. Using dual polarimetric data, only a portion of the target space can be explored and the target behavior in the rest of the space generally cannot be retrieved. For this reason, in order to obtain optimal results, it is strongly suggested to exploit the detector with quad polarimetric data. 
However, in case only dual polarimetric data are available, the algorithm can still be executed as we now show.

The final formal expression of the detector does not suffer significant changes:

$$
\gamma_{d}=\frac{1}{\sqrt{1+\operatorname{RedR}\left(\frac{P_{t o t}}{P_{T}}-1\right)}}=\frac{1}{\sqrt{1+\frac{b^{2}}{1-2 b^{2}}\left(\frac{\underline{d}^{*} \underline{d}^{*}}{\left|\underline{d}^{* T} \underline{\hat{d}}_{T}\right|^{2}}-1\right)}} \geq T
$$

where the $d$ vector is the dual polarimetric counterpart of $t$ :

$$
\begin{aligned}
\underline{d} & =\operatorname{Trace}\left(\left[C_{d}\right] \Psi_{d}\right)=\left[t_{1}, t_{2}, t_{3}\right]^{T}= \\
& =\left[\left\langle\left|k_{1}\right|^{2}\right\rangle,\left\langle\left|k_{2}\right|^{2}\right\rangle,\left\langle k_{1}^{*} k_{2}\right\rangle\right]^{T} .
\end{aligned}
$$

$\left[C_{d}\right]$ is a $2 \times 2$ coherency matrix calculated starting from the 2 dimensional complex scattering vector for dual polarimetric data.

\section{ClASSIFIER}

\section{A. Formulation}

A classifier can be designed starting from the partial target detector, where any class (i.e. partial target) is described by a specific covariance matrix $\left[C_{i}\right]$. The proposed partial target detector is exploited to generate several masks for the specific classes. If only few areas are of interest (e.g. different states of sea ice) a small number of classes are sufficient (the extreme scenario is with one single detection mask). Otherwise, several covariance matrices must be taken into account. The classification output is similar to the supervised Wishart approach [16, 30].

The detections of the classes are performed in series generating a stack of masks:

$$
\left\{\begin{array}{c}
m_{i}(x, y)=0 \quad \text { if } \gamma_{i}(x, y)<T \\
m_{i}(x, y)=\gamma_{i}(x, y) \quad \text { if } \gamma_{i}(x, y) \geq T
\end{array}\right.
$$

where $i=1, \ldots, n$ indicates the respective class.

The selection of the $S C R$ (or equivalently the threshold) has the purpose of generating the class of unknown targets. In case that the class of unknown targets is not required, the threshold of the detectors can be eliminated (or set to zero). In this case, the discrimination among classes is exclusively performed on the base of the magnitude of $\gamma_{d}$.

Subsequently, the mask with the maximum value is selected for each pixel. The normalized inner product returning the higher value is the one with the smallest angular distance to the regarded class. If $m_{1}, \ldots, m_{n}$ are the $n$ obtained masks, a pixel is allocated to the class $\mathrm{Y}$ if:

$m_{Y}=\max _{i=1, \ldots, n}\left\{m_{i}\right\}$.

In an actual implementation of the classifier, the partial target detector is executed $n$ times one after the other. In any execution, the vector representing the specific class is selected. The classifier is completed by a simple algorithm which pixel by pixel selects the mask presenting the maximum value. The classifier does not require iterations, since it converges after the first attempt.

\section{B. Parameters selection}

A straightforward strategy could be to simply use the same parameters exploited for standard detection. However, we believe the selection of $S C R=15$ reveals a significant advantage. As shown by (53), the classifier decision rule is based on the comparison of different masks and selection of the maximum. In this way, the algorithm assigns the pixel to the class with a characteristic vector closer to the observed one. With a lower $S C R$ (i.e. lower selectivity), we are able to detect observed targets presenting some slight dissimilarity from the class characteristic vector. For instance, the dense forest class should include a relatively large collection of volumes (e.g. clouds of particles with different shapes). Clearly, when the difference is too large, a new class must be introduced.

As a general consideration, in the classifier architecture, the use of a detection threshold is exclusively related to the rejection of unknown targets. In case this is not required, we could choose $S C R=0$ (which corresponds to $T=0$ ) and the discrimination would be performed only by the maximum selection (53).

\section{Supervised and Unsupervised versions}

Depending on the strategy exploited to extract the class coherency matrix, the classifier can be supervised or unsupervised.

The supervised version requires the user interaction for the selection of known areas. This operation can be easily accomplished on a RGB Pauli composite image.

The unsupervised version trains the detector exploiting polarimetric scattering models. A large assortment of models was developed in the past [1]. Considering the proposed algorithm represents a general geometrical operation on polarimetric data, any model can be equally exploited. Therefore, it is left to the user to select the most appropriate model for the particular application of interest. We present examples of both supervised and unsupervised detection and classification in section V.

\section{VALIDATION}

\section{A. Datasets employed}

In order to provide a large validation of the detector, several datasets with different settings and scenarios were employed.

The first quad polarimetric dataset was acquired by the ESAR airborne system of DLR (German Aerospace Agency) during the SARTOM campaign (Landsberg, Germany) in 2006 [31]. One aim of the campaign was target detection under foliage, for this reason several manmade targets were deployed on open field and under forest canopy cover. The frequency band is $\mathrm{L}$ and the image has a spatial resolution of 
$1.1 \mathrm{~m}$ in azimuth and about $2 \mathrm{~m}$ in range.

Subsequently, a quad-polarimetric L-band ALOS-PALSAR dataset is exploited for the detection of distributed targets. In particular, we consider detection of historical firescars based on their depolarization behavior. The images were acquired in Canada close to the town of Manning, Alberta and present a mix of agricultural and forested areas. The pixel size of ALOS quad polarimetric data is around $24 \mathrm{~m} \times 4.5 \mathrm{~m}$ (ground range $\mathrm{x}$ azimuth). In order to process the images an initial multilook of 1 x 5 (range $x$ azimuth) was followed by a boxcar averaging of $9 \times 9$. The latter led to a final equivalent number of looks (ENL) equal to 253.

Moreover, another quad-polarimetric L-band ALOSPALSAR scene is exploited for a further investigation of landuse classification. The latter was acquired in China in May 2008, close to the city of Taian and the mountain of Culai and represents a mixed urban, agricultural and mountain forest sites.

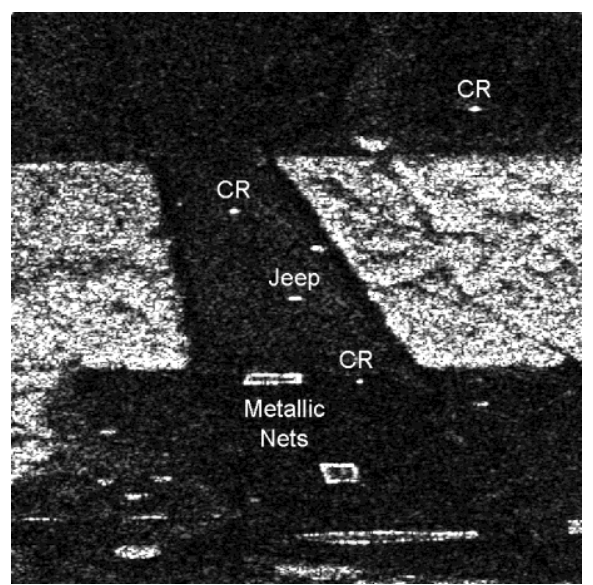

(a) HH polarization

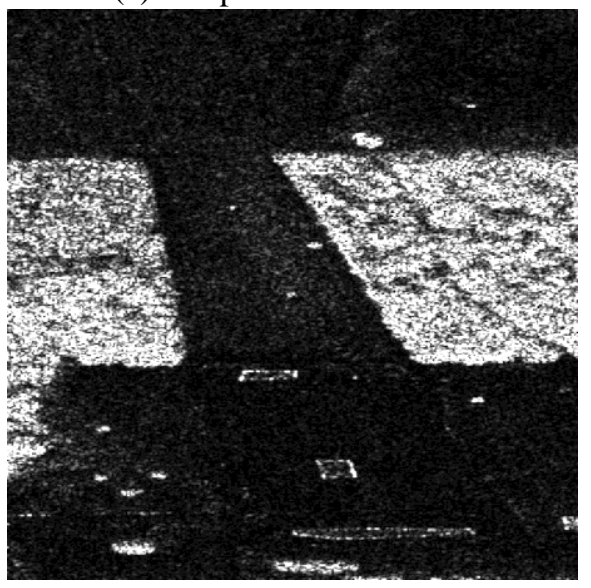

(d) HV polarization

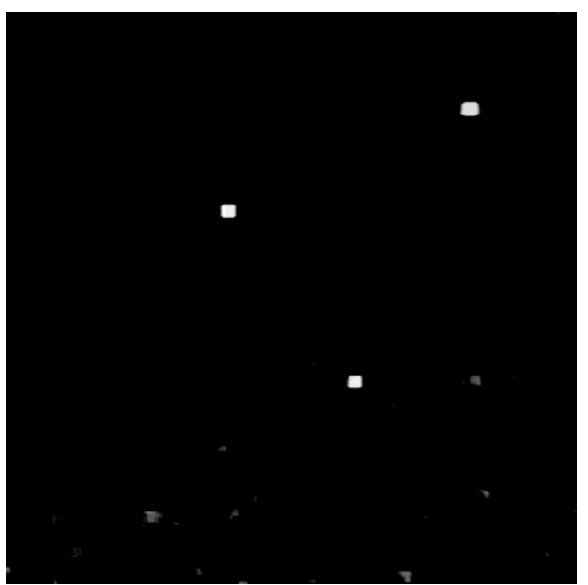

(b) STD: odd bounce

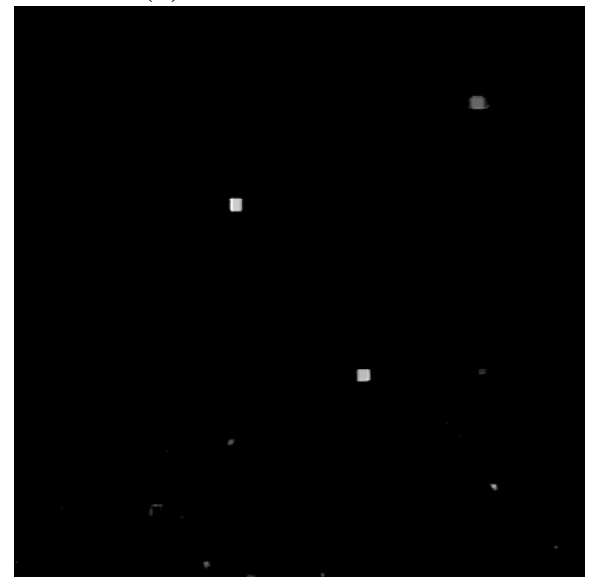

(e) PTD: odd bounce

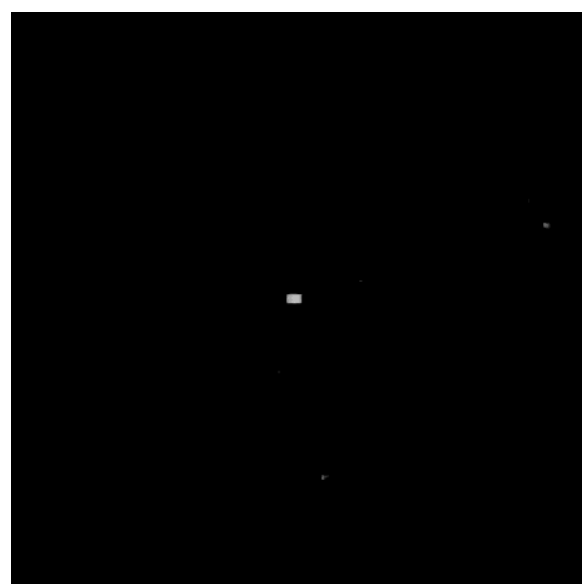

(c) STD: even bounce

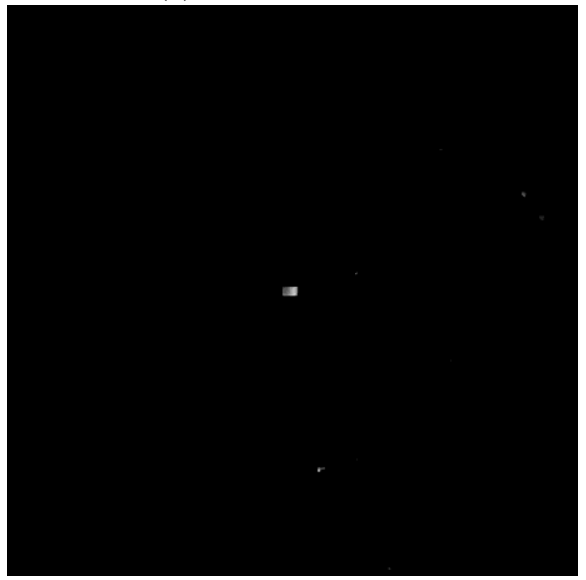

(f) PTD: even bounce

Figure 1 Comparison of detection for single and partial targets. (a) HH image with markers for relevant targets; (b) Single target detector (STD): odd bounces; (c) STD: even bounces; (d) HV image with markers for relevant targets; (e) Partial target detector (PTD): odd bounces; (f) PTD: even bounces; CR: Trihedral Corner Reflector. (ESAR L-band, DLR, Landsberg, Germany, September 2006)

The last dataset used is a TerraSAR-X Stripmap dual polarimetric HH/VV acquisition. The represented scene is again Taian in China and the data were acquired in March 2009. The resolution of the sensor is $1.2 \mathrm{~m} \times 6.6 \mathrm{~m}$ (range $\mathrm{x}$ azimuth), however the pixel dimension is about $0.9 \mathrm{~m} \times 2.4 \mathrm{~m}$. In order to process the images we performed an initial multi look of 2 by 3 (range $x$ azimuth) and a following average of 9 $\mathrm{x}$ 9. This led to a final ENL equal to 136 .

With the intention of testing different modalities of the proposed algorithm, the validation is subdivided in separate sections.

\section{A. Comparison between single and partial target detector}

Firstly, the ability to detect single targets is examined. The new algorithm is compared with the single target detector (already validated in [19-21]). Single targets represent a subspace of the partial targets, described by rank one covariance matrices [32]. Therefore they are also detectable by the new partial target detector.

Although the detectors can be used to find an arbitrary rank-1 matrix, here as an example we consider the simpler problem of detecting scattering mechanisms represented by 
the first two axes of the Pauli basis, i.e. with scattering matrices given by $\operatorname{diag}(1,1)$ and $\operatorname{diag}(1,-1)$. These targets are

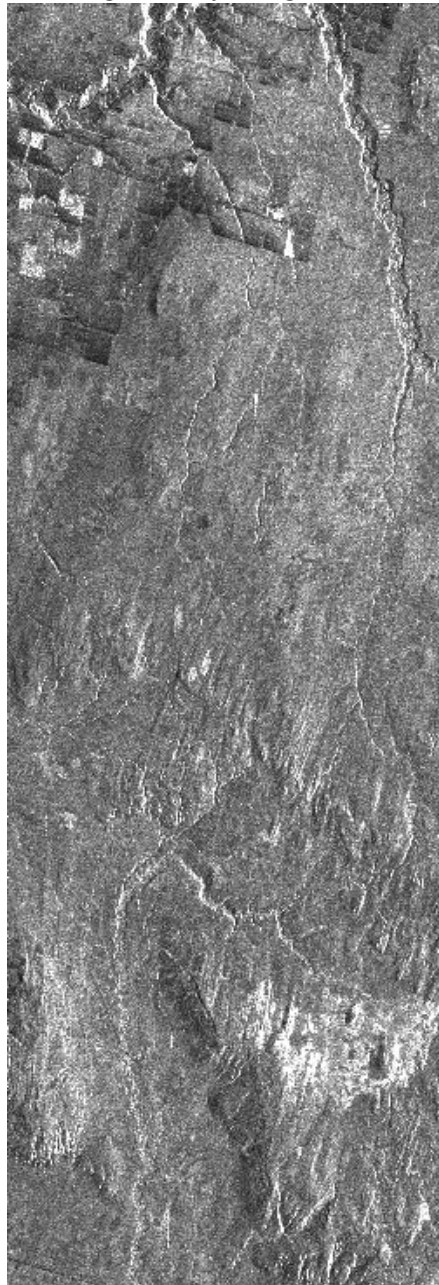

(a) First Pauli component

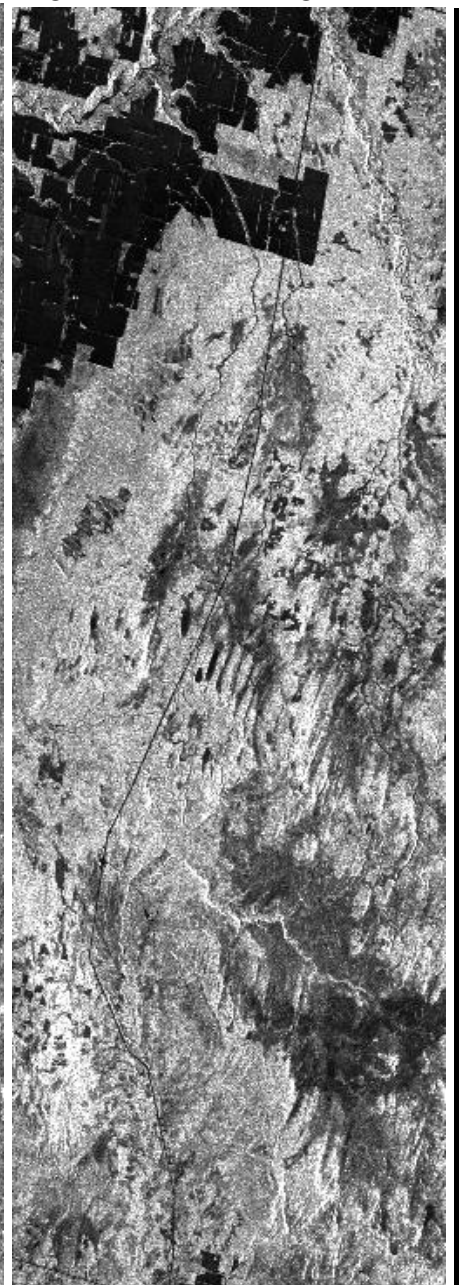

(b) Third Pauli component

more commonly known as odd and even-bounce respectively.

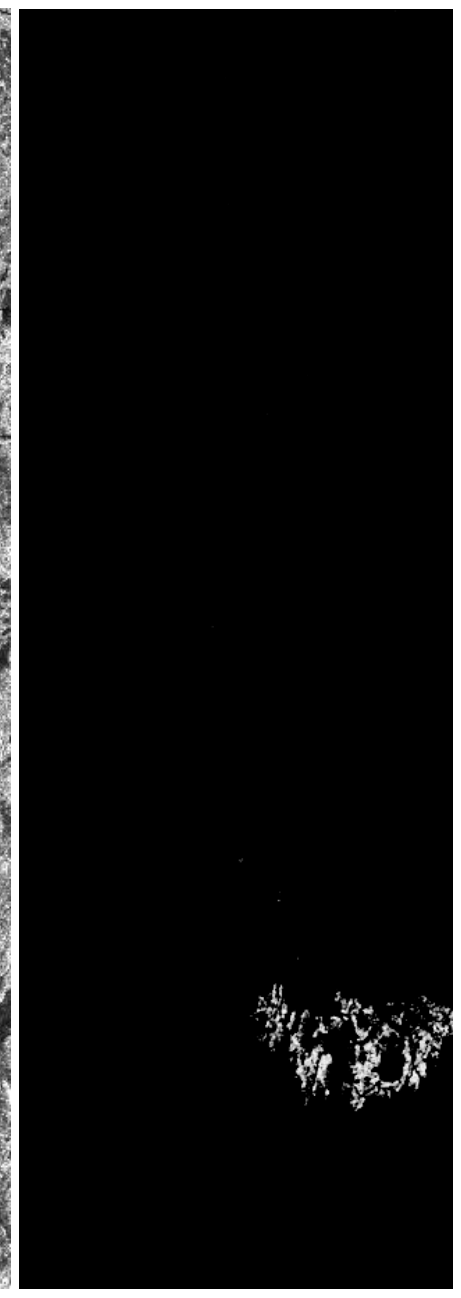

(c) PTD: Supervised

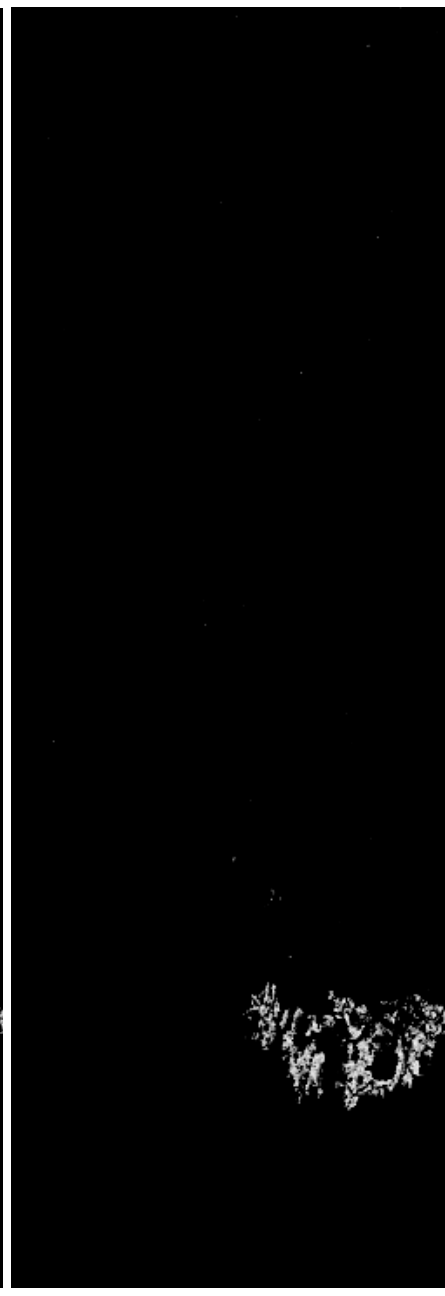

(d) PTD: Unsupervised

Figure 2 Partial target detector of ancient fire scar. (a) First Pauli component, HH+VV; (b) Third Pauli component, $2 * H V$; (c) supervised detection; (d) unsupervised detection. (ALOS-PALSAR, JAXA, Keg River, Canada, June 2009)

In point target detection a high resolution dataset is favorable, therefore the DLR L-band dataset is employed [31]. Figure 1 presents the comparison between the single and partial target detectors. The amplitudes at $H H$ and $H V$ polarization are presented as comparison. The two algorithms perform similarly, but the resulting masks are not exactly equal. More information is added in the new detector (i.e. the second order statistics of $k$ ) hence slightly better outcomes are expected (i.e. lower false alarm and missed detection rate). The mask for even-bounces (even number of reflections) identifies mainly the jeep in the middle of the scene, since it generates a horizontal dihedral with the ground surface. Moreover it is possible to recognize some trunk-ground double-bounces, especially on the edge of the forest and on a clearing, where the wave attenuation due to the canopy is less significant. The masks of odd-bounces (odd number of reflections) reveal the trihedral corner reflectors and some weaker points on the bare ground. The metallic nets are rejected since they resemble horizontal dipoles (as illustrated in [19-21]). The capability to reject bright targets is an indicator that the discrimination is based on the polarimetric information and not the intensity of the return.

\section{A. Satellite data: historical fire scar ( $h f s)$ detection}

This section is concerned with the exploitation of satellite radar data. The latter are particularly important for the scientific community and end users since they provide periodical coverage of large areas.

In this section, a quad polarimetric ALOS-PALSAR dataset will be used. Figure 2.a and Figure 2.b illustrate respectively the first and third components of the Pauli scattering vector (i.e. $H H+V V$ and $2 * H V$ ) of a scene acquired in Canada and presenting a combination of agricultural fields (up left corner) and forests. Considering that the rectangular shape of the pixel introduces severe visual distortions in the image, the data were multi-looked using an asymmetric window size of $1 \times 5$.

The multi-look was accomplished on the covariance matrix $[C]$ with the intention of preserving the polarimetric 
information [33]. The detector uses a subsequent window average of $9 \times 9$ in order to minimize speckle and accurately characterize depolarized targets in the scene [34].

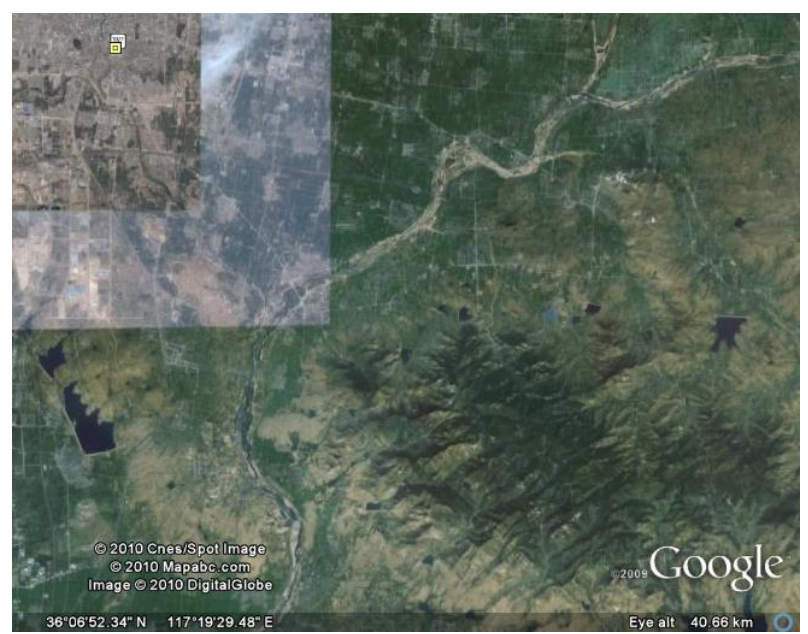

(a) RGB Pauli

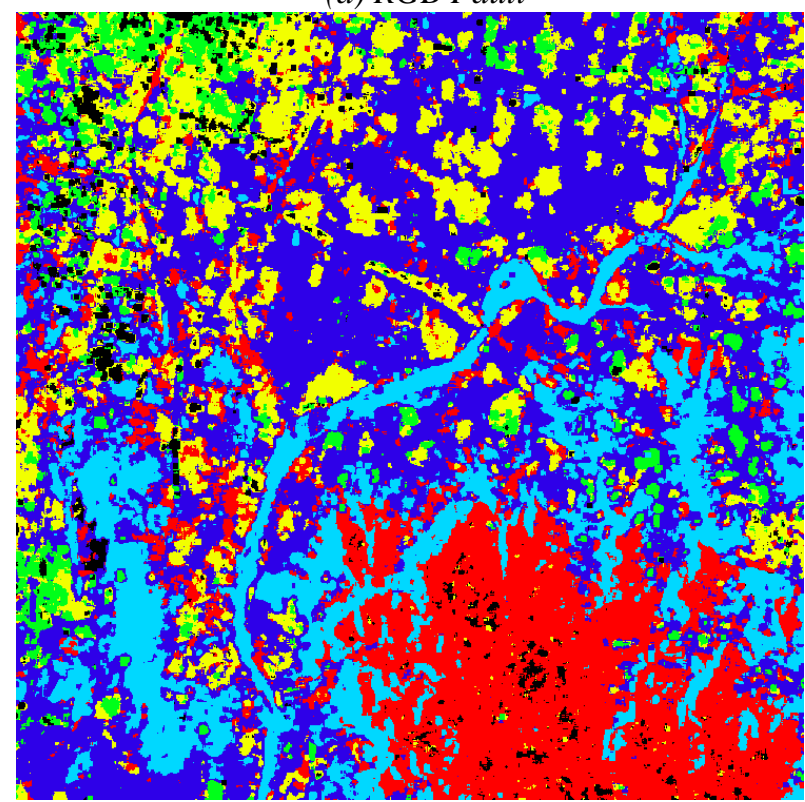

(c) PTD supervised (b) Google Earth photograph

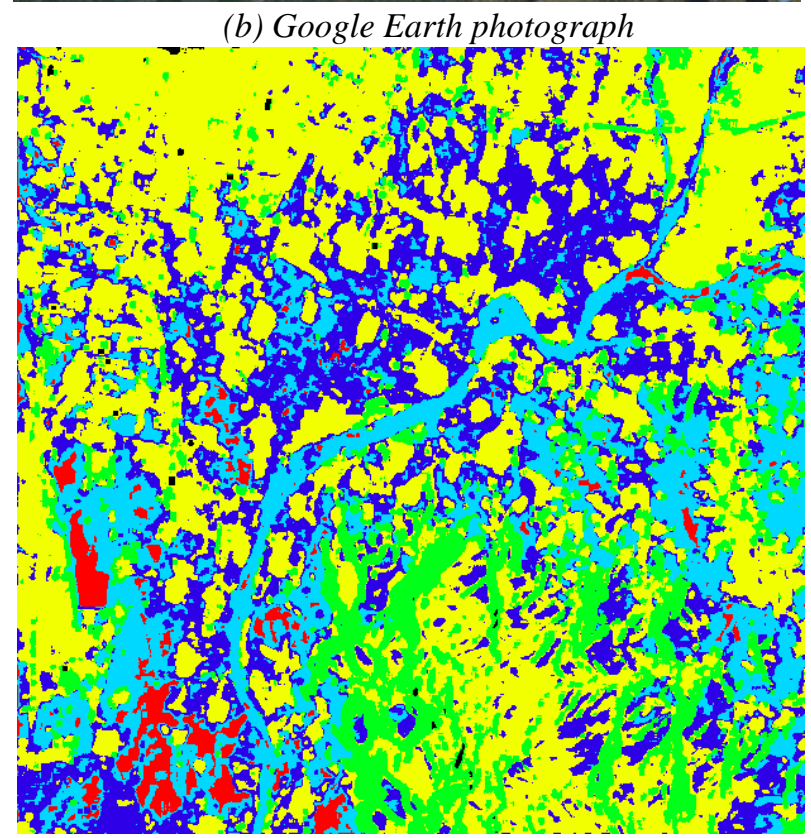

(d) Wishart supervised

Figure 3. Partial target detection on ALOS data (China): (a) RGB Pauli image of the area (b) Google Earth photograph of the scene (c) supervised classification after the detection of 4 partial targets (d) Wishart supervised classification (same classes as before). Red: dense forest; Light blu: surfaces; Blue: agricultural; Yellow: villages; Green: urban area. (ALOS-PALSAR, JAXA, Taian, China, May 2008)

The test area includes a forest region subject to a fire in 2002 (close to the bottom right corner). The historical fire scar $(h f s)$ presents structural differences with the old one due to the younger age of the trees and the absence of understory. Figure 2.c depicts the detection mask when the algorithm is trained with pixels marked as $h f s$ by the ground surveillance. The training process consisted in extracting the $\mathrm{t}$ vector for a small portion (13x65 pixels) of the fire scar and utilizing it in the following detection.

The detector reveals the capability to separate the $h f s$ from the rest of the scene, with very low false alarms rate. The subsequent step considers the examination of a forest model able to link the presence of an $h f s$ with some key parameter. The exploited model is the RVoG (Random Volume over Ground) [35, 36], where the return from the forest is described by random volume scattering plus a coherent component. The 
latter is commonly generated by the ground beneath the canopy and is described by a rank one coherency matrix (since it is a single target). The volume contribution is modeled as scattering from dipoles randomly oriented:

$$
\begin{aligned}
& {[T]=\left[T_{S}\right]+\left[T_{V}\right],} \\
& {\left[T_{S}\right]=m_{S}\left[\begin{array}{ccc}
\cos ^{2}(\alpha) & \cos (\alpha) \sin (\alpha) e^{j \mu} & 0 \\
\cos (\alpha) \sin (\alpha) e^{-j \mu} & \sin ^{2}(\alpha) & 0 \\
0 & 0 & 0
\end{array}\right],(54)}
\end{aligned}
$$$$
\left[T_{V}\right]=m_{V}\left[\begin{array}{ccc}
2 & 0 & 0 \\
0 & 1 & 0 \\
0 & 0 & 1
\end{array}\right] \text {. }
$$

Where $[T]$ is the covariance matrix expressed in the Pauli basis (also referred as coherency matrix) $m_{S}$ and $m_{V}$ are the magnitudes of the two backscattering contributions, and $\mu$ is the phase difference between first and second elements of the Pauli basis.

Their ratio is the ground-to-volume ratio

$$
\rho=\frac{m_{S}}{m_{V}} \text {. }
$$

$\alpha$ is the characteristic angle with the same meaning as in the eigenvector decomposition of the coherency matrix $[T]$ [1].

In this experiment, we exploited a model in absence of slopes, since the DEM (Digital Elevation Model) of the image is particularly flat, however, in case of relevant topography a preliminary slope correction should be accomplished [37]. In order to find the initial values for the model parameters which fit the $h f s$, the model was inverted on the data. The resulting parameters were found to be:

$\alpha=19^{\circ}$ and $\rho=7.7 \mathrm{~dB}$.

(similar results, especially regarding $\alpha$, were found for other $h f s$ in Canada). The extracted values were used to reconstruct a $[T]$ matrix to train the detector (Figure 2.d).

The model seems to approximate adequately the typology of target, since a bad fit would not allow a correct reconstruction of $[T]$. The latter is an example of exploiting a model to train the unsupervised detector, however different models can be employed, such as the oriented volume over ground (OVOG) or other multi-layer decompositions [1].

\section{A. Satellite data: classification}

In this section the algorithm is evolved into a classifier and tested over a second L-band ALOS-PALSAR dataset in China. The city of Taian (upper left corner) and the mountain of Culai (lower right corner) are clearly visible in the RGB Pauli composite image (Figure 3.a, where 1200x1200 pixels are visualized here).

Figure 3 presents the classification mask compared with the Wishart supervised $[14,16]$. The latter is a classifier exploiting an assumed a priori probability distribution of the coherency matrix $[T][1,2,14]$. In this comparison, a basic version of the Wishart supervised classifier was utilized. This is freely available in the software package POLSARpro.

We are conscious that more elaborated versions employing supplemental pre-processing can result in more accurate classification masks. However, in order to make the comparison as fair as possible, the two classifiers had exactly the same pre-processing and they both are executed in the most basic version. The absence of corrections or further processing should allow us to evaluate anticipated theoretical advantages.

In Figure 3.a, labels identify the training areas. Area1 represents agricultural fields (blue), Area2 is surfaces (light blue), Area3 is urban area (green), Area4 is a village characterized by small structures and sparse trees (yellow) and Area5 is a dense forest (red). The proposed classification has a total of 6 classes, since the black color is reserved to areas not falling in any class (i.e. unknown targets). Performing a preliminary detection (setting $S C R=15$ ) of the different typologies, the areas are not forced to adhere to any class avoiding misclassification.

The proposed algorithm seems able to separate the different areas in the scene showing significant agreement with the RGB Pauli image and the Google Earth photograph. Please note, as in the previous case, the coherency matrix is multilooked $1 \times 5$, however the pixel is not completely square and a distortion of the radar image is still visible. Moreover, the azimuth is not perfectly aligned with the north-south direction. The urban area presents an interesting scenario. The classification mask presents a conspicuous heterogeneity (due to the natural heterogeneity of the city). Specifically, there are several point targets which do not fall in any class and are separated in black. Additionally, the suburban areas resemble more the villages (yellow), rather than the dense city area.

The supervised Wishart classifier (statistical based) $[14,16]$ seems to have an overall agreement with the proposed algorithm for two classes: bare surface and agricultural fields. On the other hand, the other areas present rather scarce agreement. Specifically, in Wishart the urban area is much more extended and confused with the villages. For instance, the upper right corner is classified as a town/village while it is an agricultural area. Moreover, the forest on the mountainous area is completely misclassified presenting a mix of village and urban areas.

From this experiment, a major advantage of the proposed classifier is noticeable: the independence on the total intensity of the backscattering. Wishart is strongly dependent on the Trace of $[T]$ in the calculation of its interclass distance. On the other hand, the independence on the overall amplitude focuses our detector exclusively on the polarimetric characteristics (relative weight of the matrix elements). Please note, if in (16) we multiply $[P]$ by a scalar factor the resulting detector does not change. For Wishart, two objects can have a small distance if their power backscattered is similar even though they present some polarimetric difference.

However, in case the overall amplitude keeps essential physical meanings for a specific target, its information can be taken into account performing a subsequent amplitude 
analysis over the obtained mask. Nevertheless, the possibility to separate the polarimetric and amplitude information is considered the most significant advantage of the proposed classifier.

Obviously, if the effect of amplitude modulation can be corrected with ancillary information (e.g. a DEM) the

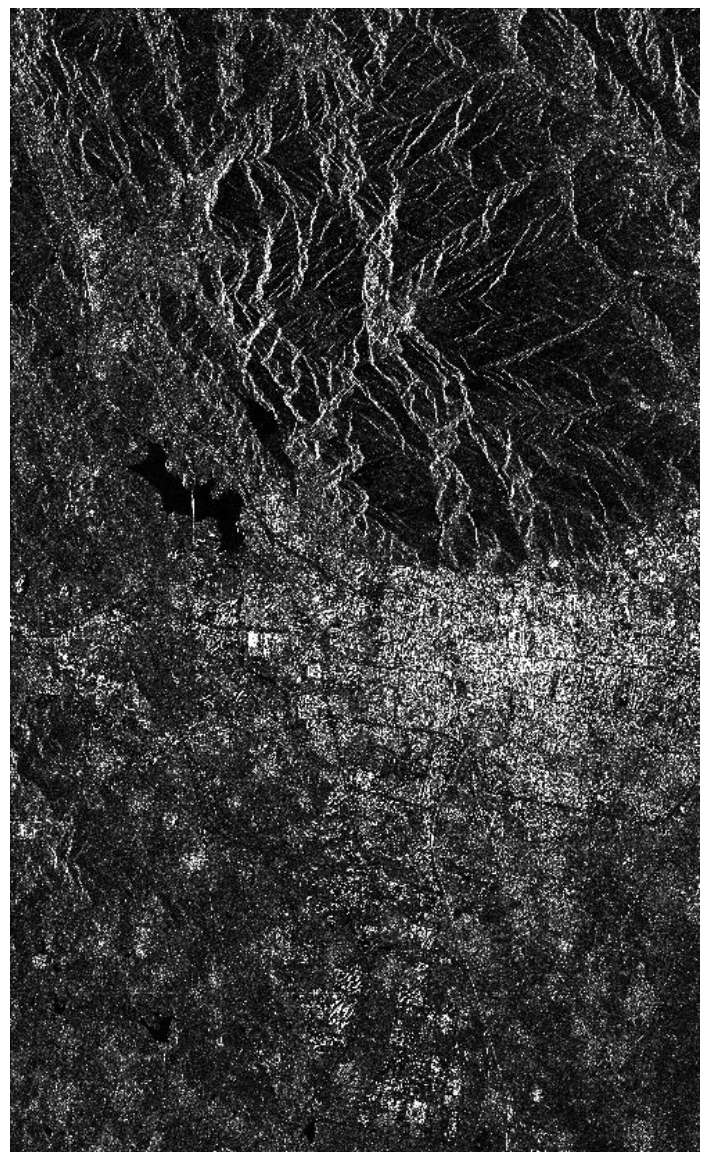

(a) $\mathrm{HH}$ reflectivity accuracy of the Wishart supervised classification mask is expected to improve, but such corrections are not always stable and robust and here we have demonstrated an approach that is not so sensitive to errors in topography compensation.

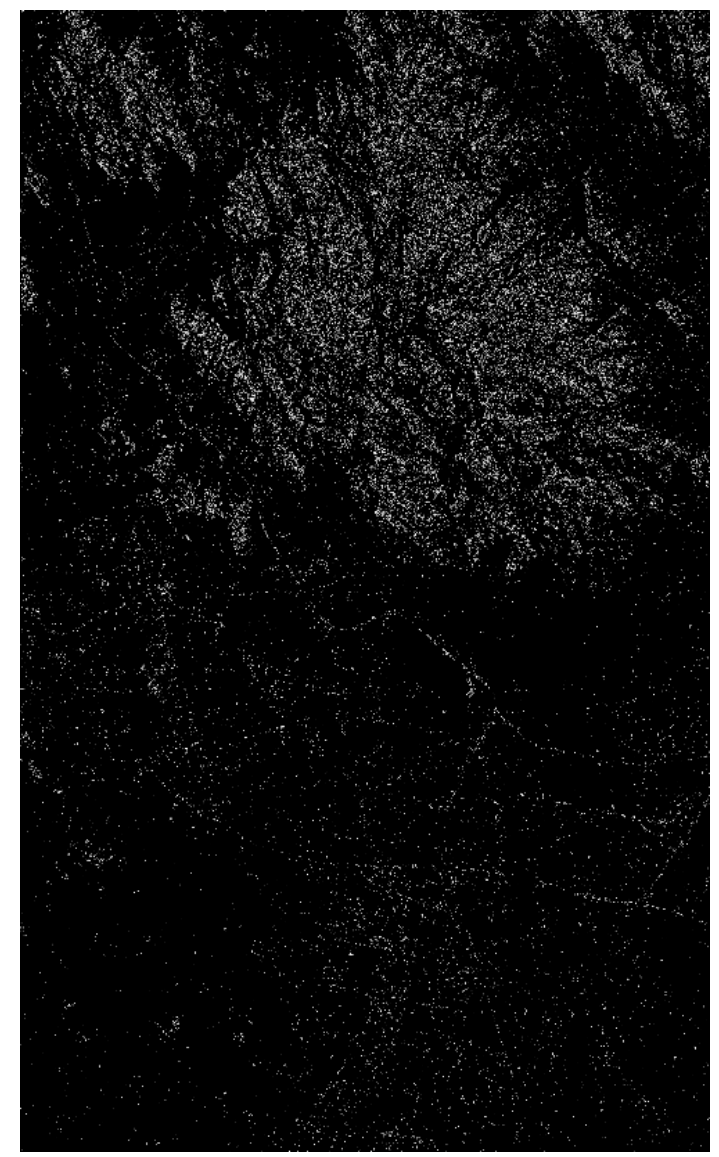

(b) Random volume detection

Figure 4. Partial target detection on TerraSAR-X dual polarimetric (HH/VV) data (China): (a) HH reflectivity image of the scene (b) detection mask of volume composed of randomly oriented dipoles. (TerraSAR-X, DLR, Taian, China, March 2009)

\section{A. Satellite data: dual polarimetric detection}

In this final experiment, the detector is tested with dual polarimetric data. The basic difference with quad polarimetric data is the lack of uniqueness in the description of an observed target [32]. For this reason, an appropriate use of the algorithm should restrict the detection to target typologies which can be represented with sufficient accuracy by only two polarisations. An example is the scattering from a random volume.

In this experiment, TerraSAR-X Stripmap dual polarimetric $\mathrm{HH} / \mathrm{VV}$ data are exploited. As for the ALOS dataset, the scene was acquired in China over the city of Taian. However, now the scene is slightly more north showing the Choushui Xuneng Reservoir (i.e. mountainous area covered by dense forests).

An initial multi-look of $2 \times 3$ (azimuth $\mathrm{x}$ range) was performed on the dual polarimetric covariance matrix. Subsequently, the detection was achieved employing a 9x9 boxcar filter. The detection is aimed at volume scattering composed of randomly oriented dipoles. In case of HH/VV dual polarimetry, we do not have direct access to a crosspolarized HV channel to detect volume scattering. Instead, the latter can be identified through its signature coherency matrix in the HH/VV subspace, expressed as shown in (57): $\left[T_{V}^{d}\right]=m_{V}\left[\begin{array}{ll}2 & 0 \\ 0 & 1\end{array}\right]$.

Figure 4 presents the detection mask (b) compared with the $H H$ reflectivity image (a). The algorithm seems able to identify the mountainous areas covered by dense forest, based on their level of volume scattering. The water reserve, in the middle left of the image, is detected since its backscattering is particularly low and close to the noise floor. Consequently, it 
resembles random volume with slightly stronger surface component. In order to remove these points, a simple threshold on the amplitude could reject areas with backscattering close to the noise floor. Regarding the detected points externally to the mountainous area, they mainly correspond to trees in the city, besides roads or around fields. Here, they are more apparent than in the ALOS data due to the enhanced resolution and the use of $\mathrm{X}$ band which is more sensitive to canopy. However, we cannot neglect that part of these points are merely false alarm due to the absence of the complete polarimetric information.

\section{CONCLUSIONS}

In this paper, a geometric interpretation has been provided for the single target detector developed in [19-21] and based on a perturbation filter. The detector is constituted by a weighted (by the observables) and normalized inner product (i.e. coherence) between the target of interest and a perturbed version. The use of a coherence was found convenient since it is a normalized entity in the closed interval 0 to 1 . Therefore the overall amplitude of the backscattering is neglected and the detection (classification) is performed exclusively on the base of the polarimetry.

In order to extend the detection to partial targets, a new vector formalism was proposed. The new formalism can describe uniquely the partial target space. Finally, the new detector was exploited as first stage of a subsequent classifier.

Validation against airborne (DLR E-SAR, L-band) and satellite data (ALOS-PALSAR and TerraSAR-X) is provided showing the capability of the detector to discriminate among different single and partial targets. The detector is an algebraic operation on a Euclidean space independent of its dimensions. Therefore, a dual polarimetric version can be developed, although we expect lower performances due to the loss of physical information. Both the supervised and unsupervised detection strategies were exploited.

The classification mask is compared with a basic Wishart supervised algorithm (freely available in the software package POLSARpro), revealing what we believe to be a major enhancement: the independence on the overall intensity of the return (i.e. the proposed algorithm works solely with the polarimetric information). Therefore, misclassifications due to modulations of the amplitude, as for example a consequence of layover, are solved, making the new algorithm particularly suited for detection and classification in mountainous regions. Clearly, if ancillary information (as a DEM) is available and further pre-processing is performed the classification result of the Wishart supervised can be significantly improved.

As a future work, the algorithm will be tested over different typologies of data (e.g. RADARSAT2) and presenting diverse scenarios (e.g. sea ice classification, ship detection, agricultural classification). Moreover, the possibility of exploiting a priori information about the clutter (i.e. hypothesis $b \neq c \neq|d| \neq|e| \neq|f|$ ) will be investigated for detection in controlled background.

\section{ACKNOWLEDGEMENT}

We would like to acknowledge partial support by the UK DSTL (in the framework of the SARTOM project run by eOsphere Ltd) and by the ESA DRAGON-2 project number 5344 (run by the University of Rennes, France). Some figures in this paper were published in the book: "Armando Marino, $A$ new target detector based on geometrical perturbation filters for polarimetric Synthetic Aperture Radar (POL-SAR), Chapter 8, Springer Verlag, Berlin, 2012. They are presented in this paper with kind permission of Springer Science+Business Media. We would like to thank the SARTOM project for the provision of the airborne E-SAR data. The ALOS PALSAR data for the fire scar detection was provided courtesy of Dr. Hao Chen and Dr. David Goodenough, Canadian Forestry Service (CFS), Victoria, BC. The ALOS-PALSAR data for the Chinese test site was provided courtesy of the DRAGON 2 program. Finally we would like to acknowledge support from TerraSAR-X project number LAN0638 for provision of the dual polarized data used.

\section{REFERENCES}

[1] S. R. Cloude, Polarisation: Applications in Remote Sensing. Oxford University Press, 978-0-19-956973-1, 2009.

[2] J. S. Lee and E. Pottier, Polarimetric radar imaging: from basics to applications. Boca Raton, 2009.

[3] L. E. Ferro-Famil, Pottier, E. , and J. S. Lee, "Unsupervised Classification of Multifrequency and Fully Polarimetric SAR Images Based on H/A/Alpha-Wishart Classifier," IEEE Transaction on Geosciance and Remote Sensing, vol. 39, pp. 2332-2342, 2001.

[4] J. S. Lee, M. R. Grunes, E. Pottier, and L. Ferro-Famil, "Unsupervised Terrain Classification Preserving Polarimetric Scattering Characteristics," IEEE Transactions on Geoscience and Remote Sensing, vol. 42, pp. 722-732, 2004.

[5] W. M. Boerner, "Basics of Radar Polarimetry," RTO SET Lecture Series, 2004.

[6] S. R. Cloude, "Group theory and polarization algebra," OPTIK, vol. 75, pp. 26-36, 1986.

[7] S. R. Cloude, "Lie Groups in EM Wave Propagation and Scattering," Chapter 2 in Electromagnetic Symmetry, Eds. C Baum, H N Kritikos, Taylor and Francis, Washington, USA, ISBN 1-56032-321-3, pp. 91142,1995

[8] S. R. Cloude and E. Pottier, "A review of target decomposition theorems in radar polarimetry," IEEE Transaction on Geoscience and Remote Sensing, vol. 34, pp. 498-518, 1996.

[9] R. D. Chaney, M. C. Bud, and L. M. Novak, "On the Performance of Polarimetric Target Detection Algorithms," Aerospace and Electronic Systems Magazine, IEEE, vol. 5, pp. 10-15, 1990.

[10] L. M. Novak, M. C. Burl, and M. W. Irving, "Optimal Polarimetric Processing for Enhanced Target Detection," IEEE Trans. Aerospace and Electronic Systems, vol. 20, pp. 234-244, 1993.

[11] L. M. Novak, S. D. Halversen, G. J. Owirka, and M. Hiett, "Effects of Polarization and Resolution on SAR ATR," IEEE Transactions on Aerospace and Electronic Systems, vol. 33, pp. 102-116, 1997.

[12] A. P. Doulgeris, S. N. Anfinsen, and T. Eltoft, "Classification With a Non-Gaussian Model for PolSAR Data," IEEE Transactions on Geoscience and Remote Sensing, vol. 46, pp. 2999 - 3009, 2008.

[13] L. Bombrun and J.-M. Beaulieu, "Fisher Distribution for Texture Modeling of Polarimetric SAR Data," IEEE Geoscience and Remote Sensing Letters, vol. 5, pp. 512 - 5, 2008.

[14] J. S. Lee, M. R. Grunes, and R. Kwok, "Classification of multi-look polarimetric SAR imagery based on the complex Wishart distribution," International Journal of Remote Sensing, vol. 15, pp. 2299-2311, 1994.

[15] L. Ferro-Famil, E. Pottier, and J. Lee, "Classification and Interpretation 
of Polarimetric SAR data," IGARSS, IEEE International Geoscience and Remote Sensing Symposium, Toronto, Canada, 2002.

[16] J. S. Lee, M. R. Grunes, T. L. Ainsworth, L. J. Du, D. L. Schuler, and S. R. Cloude, "Unsupervised Classification Using Polarimetric Decomposition and the Complex Wishart Classifier," IEEE Transaction on Geosciences and Remote Sensing, vol. 37, pp. 2249-2258, 1999.

[17] M. Jager, M. Neumann, S. Guillaso, and A. Reigber, "A Self-Initializing PolInSAR Classifier Using Interferometric Phase Differences," IEEE Transactions on Geoscience and Remote Sensing, vol. 45, pp. 3503 $3518,2007$.

[18] J. C. Curlander and R. N. McDonough, Synthetic aperture radar: systems and signal processing: Wiley, 1991.

[19] A. Marino, S. Cloude, and I. H. Woodhouse, "Polarimetric Target Detector by the Use of the Polarisation Fork," Proceedings of 4th ESA international workshop, POLInSAR 2009, 2009.

[20] A. Marino, S. R. Cloude, and I. H. Woodhouse, "A Polarimetric Target Detector Using the Huynen Fork," IEEE Transaction on Geosciences and Remote Sensing, vol. 48, pp. 2357-2366, 2010.

[21] A. Marino and I. H. Woodhouse, "Selectable Target Detector Using the Polarization Fork," IEEE Int. Geos and RS Symp. IGARSS 20092009.

[22] J. R. Huynen, "Phenomenological theory of radar targets," vol. Ph.D. Delft: Technical University The Netherlands, 1970.

[23] S. R. Cloude and E. Pottier, "An Entropy Based Classification Scheme for Land Applications of Polarimetric SAR," IEEE Transaction on Geosciance and Remote Sensing, vol. 35, pp. 68-78, 1997.

[24] H. E. Rose, Linear Algebra: a Pure Mathematical Approach. Berlin: Birkhauser, 2002.

[25] G. Strang, "Linear Algebra and its Applications," Third ed: Thomson Learning, 1988.

[26] J. S. Lee, "Speckle suppression and analysis for synthetic aperture radar images," SPIE Optical Engineering, vol. 25, pp. 636-643, 1986.

[27] C. López-Martínez and X. Fàbregas, "Polarimetric SAR Speckle Noise Model," IEEE Trans. Geoscience and Remote Sensing, vol. 41, pp. 2232- 2242, 2003.

[28] C. Oliver and S. Quegan, Understanding Synthetic Aperture Radar Images Artech House, 1998.

[29] R. Touzi, A. Lopes, J. Bruniquel, and P. W. Vachon, "Coherence estimation for SAR imagery," IEEE Transaction on Geosciences and Remote Sensing vol. 37, pp. 135-149, 1999.

[30] S. R. Cloude and E. Pottier, "An Entropy Based Classification Scheme for Land Applications of Polarimetric SAR," IEEE Transaction on Geosciance and Remote Sensing, vol. 35, pp. 68-78, 1997.

[31] R. Horn, M. Nannini, and M. Keller, "SARTOM Airborne Campaign 2006: Data Acquisition Report," DLR-HR-SARTOM-TR-001 December 2006.

[32] R. S. Cloude, "Uniqueness of target decomposition theorems in radar polarimetry," Direct and Inverse Methods in Radar Polarimetry, pp. 267296, 1992.

[33] J. S. Lee, M. R. Grunes, and W. M. Boerner, "Polarimetric Property Preserving in SAR Speckle Filtering," Proceedings of SPIE, San Diego, vol. 3120, pp. 236-242, 1997.

[34] J. S. Lee, I. Jurkevich, P. Dewaele, P. Wambacq, and A. Oosterlinck, "Speckle Filtering of Synthetic Aperture Radar Images: A Review," Remote Sensing Review, vol. 8, pp. 313-340, 1994.

[35] S. R. Cloude and K. Papathanassiou, "Polarimetric SAR Interferometry," IEEE Transaction on Geoscience and Remote Sensing, vol. 36, pp. 1551-1565, 1998.

[36] R.N. Treuhaft and R. S. Cloude, "The structure of oriented vegetation from polarimetric interferometry," IEEE Transactions on Geoscience and Remote Sensing, vol. 37, pp. 2620-2624, 1999.

[37] J. S. Lee, D. L. Schuler, T. L. Ainsworth, E. Krogager, D. Kasilingam, and W.-M. Boerner, "On the Estimation of Radar Polarization Orientation Shifts Induced by Terrain Slopes," IEEE Transactions on Geoscience and Remote Sensing, vol. 40, pp. 30-41, 2002r

Armando Marino received the MSc in Telecommunication Engineering, Universita' di Napoli "Federico II" in 2006. In 2006, he joined the High Frequency and Radar Systems Department (HR), German Aerospace Centre (DLR), Oberpfaffenhofen, where he developed his MSc thesis, which focused on SAR multi pass retrieval of forest parameters. In 2011, he was awarded the $\mathrm{Ph} . \mathrm{D}$. degree at the University of Edinburgh (School of Geosciences), Edinburgh, UK, in the field of polarimetric SAR interferomery. His PhD thesis was awarded "Best PhD Thesis 2011" by the RSPSoc (Remote Sensing and Photogrammetry society) and "outstanding PhD Thesis" by Springer Verlag, which published the thesis in 2012. Since March 2011 to October 2011, he was working with the University of Alicante, Institute of Computing Research, Spain. Currently, he is a postdoc researcher in ETH Zürich, Institute of Environmental Engineering, Switzerland.

His main research interests are target detection, classification and retrieval of biophysical parameters with polarimetric and interferometric SAR.

Shane R. Cloude (M'87-SM'96-F'01) received the B.Sc. degree from the University of Dundee, Dundee, U.K., in 1981, and the Ph.D. degree from the University of Birmingham, Birmingham, U.K., in 1987. He was a Radar Scientist at the Royal Signals and Radar Establishment (RSRE), Great Malvern, U.K., until 1987. Following this, he held teaching and research posts at the University of Dundee, the University of York, U.K., and the University of Nantes, France, before taking on his present role in 1996. He is currently Director and Senior Scientist with Applied Electromagnetic Consultants (AELc), Edinburgh, U.K., undertaking contract research on a range of problems associated with radar remote sensing. Dr Cloude is a Fellow of the IEEE, the Alexander von Humboldt Society in Germany.

Iain H. Woodhouse Iain H. Woodhouse is a Senior Lecturer in Radar Remote Sensing at the School of GeoSciences, University of Edinburgh. His expertise is in the retrieval of biophysical properties of vegetation using active remote sensing, specifically synthetic aperture radar (SAR) and lidar. A key focus of his approach is the linking of observational models with process models. He is a principal investigator on a number of projects related to quantitative measurements of forests from remote sensing, funded by NERC, DTI, DSTL and the Forestry Commission. He is also a member of the UK National Space Technology Steering Group, a Director of cArbomap Ltd and a Non-executive Director of Ecometrica Ltd. He was a founding member of the Edinburgh Earth Observatory (EEO), a Research Group within the School of GeoSciences. Iain has a PhD from the Heriot-Watt University, an MSc in Remote Sensing from Dundee University and a BSc(hons) from the University of Edinburgh. He has also worked at the Marconi Research Centre and Wageningen University. In 1999 he moved to the University of Edinburgh. 